4. Monecke S, Slickers P, Gawlik D, Müller E, Reissig A, Ruppelt-Lorz A, et al. Variability of SCCmec elements in livestockassociated CC398 MRSA. Vet Microbiol. 2018;217:36-46. http://dx.doi.org/10.1016/j.vetmic.2018.02.024

5. Kadlec K, Fessler AT, Hauschild T, Schwarz S. Novel and uncommon antimicrobial resistance genes in livestock-associated methicillin-resistant Staphylococcus aureus. Clin Microbiol Infect. 2012;18:745-55. http://dx.doi.org/10.1111/j.1469-0691. 2012.03842.x

6. Price LB, Stegger M, Hasman H, Aziz M, Larsen J, Andersen PS, et al. Staphylococcus aureus CC398: host adaptation and emergence of methicillin resistance in livestock. MBio. 2012; 3:e00305-11. http://dx.doi.org/10.1128/mBio.00305-11

7. He L, Zheng HX, Wang Y, Le KY, Liu Q, Shang J, et al. Detection and analysis of methicillin-resistant human-adapted sequence type 398 allows insight into community-associated methicillin-resistant Staphylococcus aureus evolution. Genome Med. 2018;10:5. http://dx.doi.org/10.1186/s13073-018-0514-9

Address for correspondence: Geoffrey W. Coombs, Murdoch University, Antimicrobial Resistance and Infectious Diseases Laboratory, School of Veterinary and Life Sciences, Murdoch, WA, Australia; email: g.coombs@murdoch.edu.au

\section{Candida auris Sternal Osteomyelitis in a Man from Kenya Visiting Australia, 2015}

\section{Christopher H. Heath, ${ }^{1}$ John R. Dyer, Stanley Pang, Geoffrey W. Coombs, Dianne J. Gardam ${ }^{1}$}

\footnotetext{
Author affiliations: Royal Perth Hospital, Perth, Western Australia, Australia (C.H. Heath); University of Western Australia, Crawley, Western Australia, Australia (C.H. Heath); Fiona Stanley Hospital, Murdoch, Western Australia, Australia (C.H. Heath, J.R. Dyer); PathWest Laboratory Medicine WA, Murdoch (C.H. Heath, S. Pang, G.W. Coombs, D.J. Gardam); Murdoch University, Murdoch (S. Pang, G.W. Coombs)
}

DOI: https://doi.org/10.3201/eid2501.181321

In Australia in 2015, Candida auris sternal osteomyelitis was diagnosed in a 65-year-old man with a history of intensive care treatment in Kenya in 2012 and without a history of cardiac surgery. The isolate was South Africa clade III. Clinicians should note that $C$. auris can cause low-grade disease years after colonization.
C andida auris, first reported in Japan in 2009 (1), is an emerging pathogen that has caused severe disease in hospitalized patients in many countries, including India, South Africa, Spain, the United Kingdom, the United States, and Venezuela (2-4). In July 2015, a 65 -year-old man from Kenya visiting Australia for the first time sought treatment in Perth, Western Australia, Australia, for chronically discharging sternal sinus persisting for $>1$ year. His active medical problems included severe hypercapneic chronic obstructive pulmonary disease with pulmonary hypertension, ischemic heart disease, and chronic kidney impairment. In July 2012, he had unstable angina treated by coronary stenting that was complicated by cardiac arrest with cardiopulmonary resuscitation, which resulted in sternal injuries and a 3 -month intensive care unit hospitalization in Nairobi, Kenya. At hospital admission, computed tomography scan of the chest showed a $3.3-\mathrm{cm}$ subcutaneous collection and bony changes from chronic sternal osteomyelitis (Figure). Surgical debridement confirmed sternal osteomyelitis with parasternal abscesses. Posaconazole was given as pragmatic oral therapy, and trough serum levels of $2.0 \mathrm{mg} / \mathrm{L}$ at week 2 and $2.60 \mathrm{mg} / \mathrm{L}$ at week 4 were achieved. The patient died from progressive cardiorespiratory failure 3 months later.

Deep operative sternal bone samples yielded a yeast on Difco CHROMagar Candida medium (Becton Dickinson, https://www.bd.com/) that did not produce pseudohyphae or germ tubes. The isolate grew well at $40^{\circ} \mathrm{C}$ and $42^{\circ} \mathrm{C}$ but not $45^{\circ} \mathrm{C}$. Matrix-assisted laser desorption/ionization timeof-flight mass spectrometry (MALDI version 3.1; Bruker Daltonics, https://www.bruker.com/) identified the pathogen as Candida auris (score $>2.1$ ).

Sequencing of the $18 \mathrm{~S}$ rDNA internal transcribed region and 28S rDNA D1-D2 regions confirmed pathogen identification (Appendix Figure 1, https://wwwnc. cdc.gov/EID/article/25/1/18-1321-App1.pdf). We edited the DNA sequences, assembled consensus sequences using SeqScape (Applied Biosystems, https://www.thermofisher.com/us/en/home/brands/applied-biosystems.html), and performed sequence alignments with BLAST (https:// blast.ncbi.nlm.nih.gov/Blast.cgi). The internal transcribed regions of our isolate matched $100 \%$ with $C$. auris reference strain KP131674.1. The D1-D2 regions of the isolate also matched $100 \%$ with those of multiple $C$. auris isolates (GenBank accession nos. JQ219331-2, KM000828, KM000830, KU321688). Susceptibility testing with the Sensititre YeastOne YO10 panel (Trek Diagnostic Systems, https://www.thermofisher.com/) showed fluconazole resistance (MIC $>256 \mathrm{mg} / \mathrm{L}$ ) and posaconazole susceptibility (MIC $0.06 \mathrm{mg} / \mathrm{L}$ ) (Appendix Table).

\footnotetext{
${ }^{1}$ These authors contributed equally to this article.
} 
We performed whole-genome sequencing (WGS) on the isolate (FSMC57608) using the NextSeq platform (Illumina, https://www.illumina.com/) and then assembled Illumina paired-end sequencing data using SPAdes, St. Petersburg genome assembler 3.1.1 (http://spades.bioinf. spbau.ru/release3.1.1/manual.html). We identified core genome single-nucleotide polymorphisms (SNPs) using Snippy version 4.0 (http://www.vicbioinformatics.com/ software.snippy.shtml), using the C. auris B8441 genome for reference and previously described methods (2), and mapped $\approx 97.77 \%$ of the reads. A maximum-parsimony phylogenetic tree was constructed by using MEGA version 7.0 (https://www.megasoftware.net/) and 10 other C. auris genomes (2). Results showed that FSMC57608 (GenBank accession no. SRP156632) is a South Africa clade III isolate (Appendix Figure 2) with SNPs V125A and F126L and wild-type at amino acid positions 132 and 143 of Erg11 (gene associated with azole class antifungal drug resistance) (Appendix Figure 3).

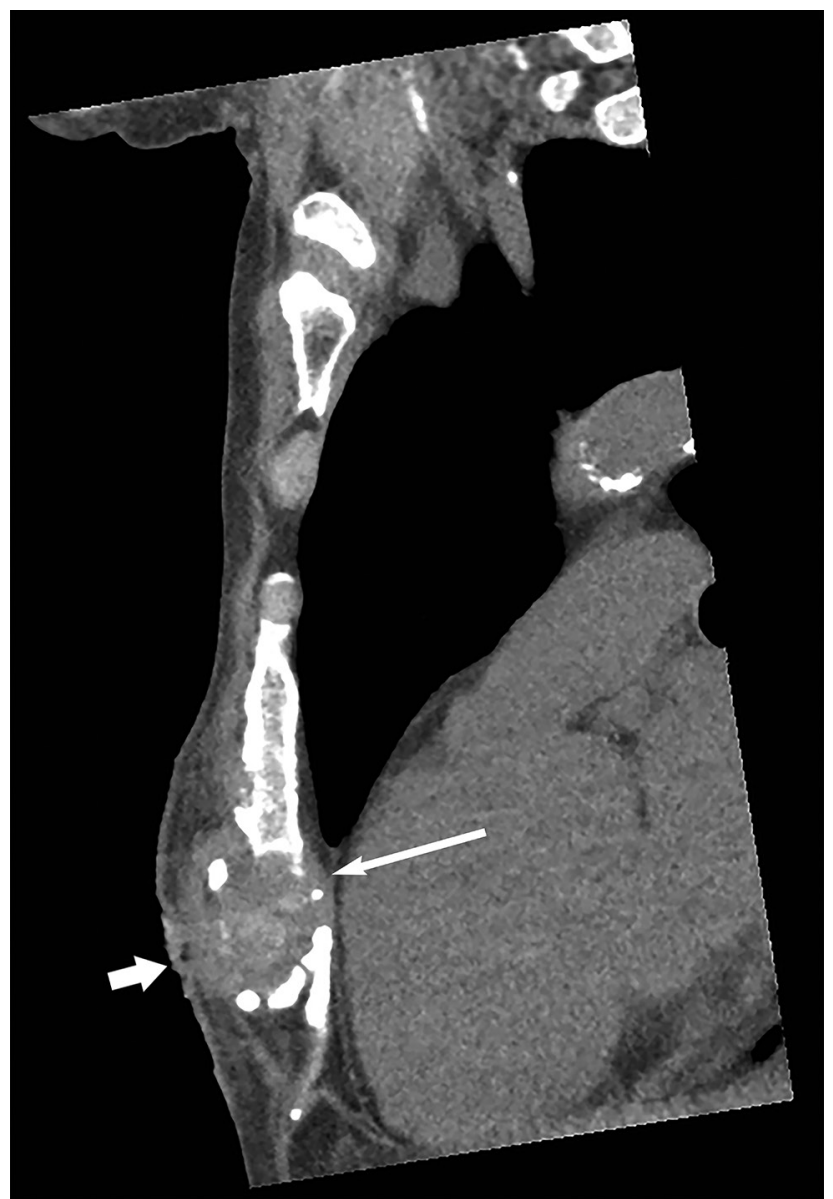

Figure. Computed tomography scan of the chest wall (sagittal section, bony windows) of man from Kenya with Candida auris sternal osteomyelitis, Australia, 2015. Image shows bony erosion and fragmentation of distal sternum (thin arrow), together with a 3.3-cm abscess and a sinus tract in the subcutaneous tissues (thick arrow).
Extensive nosocomial transmission of $C$. auris has been documented, and mortality rates of $40 \%-60 \%$ have been reported for patients with candidemia (2-4). C. auris can colonize human skin for months $(5,6)$. Of 620 cases of $C$. auris infection linked to outbreaks in Europe during 2013-2017, a total of 466 (75.2\%) patients became colonized (3). We postulate that our patient became colonized in 2012 in an intensive care unit in Kenya. This case also illustrates that clinical manifestations of $C$. auris infection can progress slowly for $>12$ months.

C. auris is multidrug resistant and, therefore, poses a risk for all patients, given the limited antifungal options available. Tentative $C$. auris-specific MIC breakpoints exist, pending further correlation between MICs and clinical outcomes (2). Proposed breakpoints are derived from expert opinion and/or those of closely related Candida species for antimicrobial drugs (e.g., amphotericin B) that do not have breakpoints. Despite breakpoint uncertainty and concerns about emergent multidrug resistance among $C$. auris isolates, we had prescribed oral posaconazole for our patient because of the in vitro MIC results and his strong preference for oral antifungal therapy.

WGS results show $C$. auris isolates fall into 4 distinct clades that appear to have emerged almost simultaneously in different geographic regions of the globe (2-4). Isolate FSMC57608 has SNPs V125A and F126L in Erg11, the latter SNP, F126L, having been described in previous investigations (J.F. Muñoz, unpub. data, https:// doi.org/10.1101/299917) (2,7). This isolate was also wild type at amino acid positions 132 and 143 of Erg11, as seen in Africa isolates (J.F. Muñoz, unpub. data, https://doi. org/10.1101/299917), further supporting that the infection originated in Africa (7).

In summary, we describe a case of travel-linked $C$. auris infection manifesting as chronic sternal osteomyelitis, diagnosed in Australia in 2015. The patient had a history of intensive care treatment in Kenya, a country with documented $C$. auris transmission (2); he required treatment in Australia 3 years later and exhibited clinically significant disease associated with South Africa clade III C. auris infection.

\section{Acknowledgments}

The authors thank the patient's next of kin for providing permission to publish this case.

\section{About the Author}

Dr. Heath is an infectious diseases physician in the Department of Infectious Diseases at Fiona Stanley Hospital and Royal Perth Hospital in Perth, Western Australia, Australia, and a clinical microbiologist for PathWest Laboratory Medicine, FSH Network, Perth; he has research interests in laboratory 
mycology and diagnostics and a special interest in infections in the immunocompromised host, particularly invasive fungal infections. Mrs. Gardam is the senior medical scientist in mycology for PathWest Laboratory Medicine; she has an active interest in diagnostics for pathogenic fungi and antifungal susceptibility testing, together with undergraduate and postgraduate education and teaching of medical mycology.

\section{References}

1. Satoh K, Makimura K, Hasumi Y, Nishiyama Y, Uchida K, Yamaguchi H. Candida auris sp. nov., a novel ascomycetous yeast isolated from the external ear canal of an inpatient in a Japanese hospital. Microbiol Immunol. 2009;53:41-4. http://dx.doi.org/10.1111/j.1348-0421.2008.00083.x

2. Lockhart SR, Etienne KA, Vallabhaneni S, Farooqi J, Chowdhary A, Govender NP, et al. Simultaneous emergence of multidrug resistant Candida auris on three continents confirmed by whole genome sequencing and epidemiological analyses. Clin Infect Dis. 2017;64:134-40. http://dx.doi.org/10.1093/cid/ciw691

3. Kohlenberg A, Struelens MJ, Monnet DL, Plachouras D; The Candida auris Survey Collaborative Group. Candida auris: epidemiological situation, laboratory capacity and preparedness in European Union and European Economic Area countries, 2013 to
2017. Euro Surveill. 2018;23. http://dx.doi.org/10.2807/1560-7917. ES.2018.23.13.18-00136

4. Chowdhary A, Sharma C, Meis JF. Candida auris: a rapidly emerging cause of hospital-acquired multidrug-resistant fungal infections globally. PLoS Pathog. 2017;13:e1006290. http://dx.doi.org/10.1371/journal.ppat.1006290

5. Centers for Disease Control and Prevention. Tracking Candida auris. 2018 Oct 3 [cited 2018 Jul 17]. https://www.cdc.gov/fungal/ candida-auris/tracking-c-auris.html

6. Welsh RM, Bentz ML, Shams A, Houston H, Lyons A, Rose LJ, et al. Survival, persistence, and isolation of the emerging multidrug-resistant pathogenic yeast Candida auris on a plastic health care surface. J Clin Microbiol. 2017;55:2996-3005. http://dx.doi.org/10.1128/JCM.00921-17

7. Rhodes J, Abdolrasouli A, Farrer RA, Cuomo CA, Aanensen DM, Armstrong-James D, et al. Genomic epidemiology of the UK outbreak of the emerging human fungal pathogen Candida auris. Emerg Microbes Infect. 2018;7:43. http://dx.doi.org/10.1038/ s41426-018-0045-x

Address for correspondence: Christopher H. Heath, Fiona Stanley Hospital, Department of Infectious Diseases, 102-118 Murdoch Dr, Murdoch, PO Box 404, Bull Creek, WA 6149, Australia; email: chris.heath@health.wa.gov.au

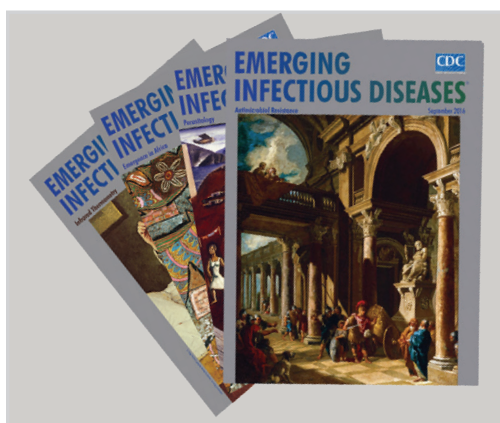

EMERGING INFECTIOUS DISEASES September 2016

- Co-Infections in Visceral Pentastomiasis, Democratic Republic of the Congo

- Multistate US Outbreak of Rapidly Growing Mycobacterial Infections Associated with Medical Tourism to the Dominican Republic, 2013-2014

- Virulence and Evolution of West Nile Virus, Australia, 1960-2012

- Phylogeographic Evidence for 2 Genetically Distinct Zoonotic Plasmodium knowlesi Parasites, Malaysia

- Hemolysis after Oral Artemisinin Combination Therapy for Uncomplicated Plasmodium falciparum Malaria

- Enterovirus D68 Infection in Children with Acute Flaccid Myelitis, Colorado, USA, 2014

- Middle East Respiratory Syndrome Coronavirus Transmission in Extended Family, Saudi Arabia, 2014

- Exposure-Specific and Age-Specific Attack Rates for Ebola Virus Disease in Ebola-Affected Households, Sierra Leone

\section{Antimicrobial resistance}

- Outbreak of Achromobacter xylosoxidans and Ochrobactrum anthropi Infections after Prostate Biopsies, France, 2014

- Human Babesiosis, Bolivia, 2013

- Assessment of Community Event-Based Surveillance for Ebola Virus Disease, Sierra Leone, 2015

- Probable Rabies Virus Transmission through Organ Transplantation, China, 2015

- Cutaneous Melioidosis Cluster Caused by Contaminated Wound Irrigation Fluid

- Possible Role of Fish and Frogs as Paratenic Hosts of Dracunculus medinensis, Chad

- Time Lags between Exanthematous Illness Attributed to Zika Virus, Guillain-Barré Syndrome, and Microcephaly, Salvador, Brazil

- Use of Unamplified RNA/cDNA-Hybrid Nanopore Sequencing for Rapid Detection and Characterization of RNA Viruses 